\title{
1 Bringing plants and soils to life through a simple role-playing activity
}

2

Michael E. Van Nuland ${ }^{1 *}$, Miranda Chen ${ }^{2}$, and Benjamin J. England ${ }^{3}$

${ }^{1}$ Department of Biology, Stanford University, Stanford, CA 94305

${ }^{2}$ University of Tennessee, Department of Ecology and Evolutionary Biology, Knoxville, TN, 37917

${ }^{3}$ University of Tennessee, Division of Biology, Knoxville, TN 37996

\begin{abstract}
Interactions are at the core of many ecological and evolutionary forces in nature. Plant-soil interactions provide a rich example of the interconnectedness of living systems, but are hidden from everyday view and overshadowed in the classroom by more popular teaching examples involving animals, reptiles, or invertebrates. To highlight the importance and relevance of plantsoil relationships, we devised a simple role-playing activity suitable for college students. Specifically, the activity simulates how feedbacks between plants and soil environments influence plant species abundance and community richness. With this activity, students will gain a better understanding of these prolific, but overlooked, forms of biological interactions that impact the diversity and functioning of ecosystems.
\end{abstract}

Keywords: diversity, feedbacks, fungi, interactions, plants, soils 


\section{Motivation}

Biological interactions are a predominant way in which students learn about the

24 ecological and evolutionary processes that influence biodiversity. However, most general

25 biology textbooks primarily use animals, reptiles, or invertebrates as case studies to demonstrate

26 the importance of interactions in nature (Uno, 1994; Schussler et al., 2010; Link-Perez et al.,

27 2010). This contrasts with the fact that (1) plants are ubiquitous, and students encounter them

28 regularly in their daily lives, and (2) most interactions that plants rely on happen belowground.

29 Since it can be difficult to present plants (and especially soils) in exciting ways, many students

30 unintentionally cultivate a fauna-centric viewpoint of the natural world (Wandersee \& Schussler,

31 2001). To highlight the importance and relevance of plant-soil relationships, we devised a simple

32 role-playing activity suitable for college students.

\section{Context}

Research on plant-soil interactions and their importance in ecology and evolution has

36 blossomed in recent decades. Specifically, feedbacks occur when plants condition soil properties

37 and, in return, are affected by the conditioned soils (Bever, 1994). Negative feedbacks reduce the

38 performance of individuals of the same species relative to other species, resulting in negative

39 frequency-dependent selection (Packer \& Clay, 2000, Mangan et al., 2010). Positive feedbacks

40 encourage conspecifics to thrive in their respective soils more than heterospecifics, leading to the

41 monodominance of single species (e.g., invasive species). These reciprocal interactions can

42 shape the non-random assembly of plant communities (Bever 1994; van der Putten 2013).

\section{Purpose}


The activity has two main purposes: (1) to engage students in a more active interpretation and discussion of the interactions between plants and soils, and (2) to connect these interactions

47 to larger concepts of drivers of biodiversity and ecosystem function. This aligns with a core

48 concept in Biology (sensu AAAS, 2011): living systems are interconnected and interacting.

49 Plant-soil interactions provide a rich example of the interconnectedness of living systems, but are

50 hidden from everyday view and overshadowed by more popular teaching examples. By actively

51 role-playing plants and soils, students can see how these interactions operate in nature.

\section{Activity description}

The primary learning objective of this activity is for students to recognize how plant-soil

interactions alter patterns of plant community diversity. This activity simulates how plant-

soil feedbacks influence species abundance and richness over time. It is recommended that the

game first be played in Negative Mode. A Positive Mode variation is introduced at the end of

this description.

Negative Mode demonstrates how negative plant-soil feedbacks promote and maintain

60 diversity. When the same plant species and soil properties are matched, the plant dies.

61 Oppositely, plants survive when species and conditioned soils are mismatched. For example, if a

62 Spruce interacts with soil that has been previously conditioned by Spruce, that plant will die. But

63 if the soil has been conditioned by Pine, Ash, or Ailanthus species, the Spruce will survive.

The directions for setup are as follows:

(1) Split the class so that there are equal numbers of plant and soil players (Fig. 1a). Clear enough space in the room so that plant and soil players can stand in two opposing lines with no obstacles between the groups (Fig. 1b). Soil players each receive one 
blank notecard and paperclip. Include a pile of paper cutouts of the different plant species ( $3 \mathrm{x}$ the total amount of plant players for each species) to serve as the species pool, as well as a separate discard pile for when plants die and are not returned to the species pool.

(2) Each plant player randomly draws one species from the species pool and returns to the line opposing soil players. Record the abundance of each plant species in the community at this initial time point $\left(\mathrm{T}_{0}\right)$. These abundances are recorded on multiple graphs (one graph for each time point; Fig. 2a). Note: the game can be purposefully set at different levels of diversity at $\mathrm{T}_{0}$.

After set-up, the gameplay begins:

(3) Plant players approach a random soil player to begin the conditioning phase that determines how plants change the physical, chemical, and biotic components of their soils based on traits related to their identity. For example, Ash leaves have lower carbon $(\mathrm{C})$ to nitrogen $(\mathrm{N})$ ratios than Spruce needles, which affects the quality of litter inputs to the soil and structures decomposer communities. To simulate conditioning, plant players hand their species to soil players, and soil players paperclip the species behind their soil card to hide the species that conditioned them.

(4) Plant players then randomly draw another species from the species pool, form a new line opposing the now-conditioned soil players, and interact with a random soil player. It is important that plant players do not know the conditioned status of soil players before interacting with them (just as tree seedlings cannot preferentially choose more hospitable soil locations in a forest). In this interaction, plants approach 
soils and show their species identity; in response, soils reveal to the plants what species they have been conditioned with. If they match, the plant dies.

(5) Plants that survive remain standing next to their respective soil player (no other plant can interact with soils that have a surviving plant). Plants that die discard their species in a separate pile (i.e., their genes do not get returned to the gene pool), before unmatched soil players. This gameplay continues until all plants are surviving and matched with a soil player. At this point, pause the game to record diversity with species' abundances (Fig. 1b).

(6) Once diversity has been recorded, plants re-condition soils with their current species identity, making soil players clip the new species over their previous species before generations $\left(\mathrm{T}_{0}-\mathrm{T}_{4}\right.$, or longer).

107 invasion success through allelopathy). To play in Positive Mode, one plant species must be 108 designated to have positive soil interactions, while all other plant species continue playing in

109 Negative Mode. This designated species survives in all soil types conditioned by all species

110 (including its own), and no other plant species can survive in soils conditioned by the designated

111 species (Fig. 1b). For example, Ailanthus is an invasive tree species that produces toxic

112 chemicals that inhibit the growth of nearby plants (Heisey 1990). Therefore, if Ailanthus were 
113 designated to have positive interactions, they would survive in any soil no matter what plant

114 species conditioned that soil. In addition, Pine, Spruce and Ash species would die if they interact

115 with Ailanthus-conditioned soil or their respective soils.

\section{Assessment, feedback, and suggestions}

$118 \quad$ For a follow-up activity, we asked students to relate the direction of plant-soil feedbacks

119 (positive or negative) to the abundance of specific plant species and total species richness (using

120 results from the primary literature; Packer \& Clay, 2000, Bais et al., 2003, Klironomos, 2002,

121 Mangan et al., 2010, Bennett et al. 2017). Students successfully predicted that species with

122 negative feedbacks would be rarer in communities that could sustain a greater number of total

123 species, and that species with positive plant-soil interactions would be more abundant in less

124 diverse communities.

125 We also asked the following question before and after the activity: "What do plant-soil

126 feedbacks make you think of?". Responses that included the words "fungi" or "mycorrhizae"

127 increased 75\%, and the word "diversity" appeared only in post-activity responses (7 out of 26

128 responses) (Fig. 2b). This suggests that students began to recognize how the nature of plant-soil

129 relationships relate to biodiversity patterns.

130 The activity takes $\sim 30$ minutes to complete and preceded a brief lecture and small group

131 work in an upper-level Ecology course (24 students, 18-25 years old). Depending on student

132 level and module topic, instructors using this activity could discuss a range of mechanisms, such

133 as soil nutrient depletion by the plant, mutualistic benefits from mycorrhizal fungi, or build-up of

134 soil-borne pathogens. Since plant-soil interactions have been explored in a variety of areas (van

135 der Putten et al. 2013), the activity can be uniquely paired with different biology topics. For 
136 introductory students, shapes could be used in place of species to focus on the mechanics of

137 feedback loops in nature.

138 This activity may be most applicable for small class sizes (20-40 students). In larger

139 classes, this activity could be implemented as a demonstration with student volunteers or during

140 discussion/laboratory sections. We found it best to use paper cutouts and notecards to drive home

141 the role-playing aspects of the game. We have provided resources for teachers to print the

142 species used in the current example (https://github.com/mvannuland/Species_supplies), but the

143 activity is amenable to any suite of species (4-5 plant species is the appropriate number for a

144 small class). With an inexpensive, time-efficient, and engaging activity, we hope to enable

145 teachers to encourage student understanding of prolific, but overlooked, forms of biological

146 interactions that impact the diversity and functioning of ecosystems. 


\section{References}

148 American Association for the Advancement of Science (AAAS). (2011). Vision and Change in Undergraduate Biology Education: A Call to Action. Washington, DC.

Bais, H. P., Vepachedu, R., Gilroy, S., Callaway, R. M., \& Vivanco J. M. (2003). Allelopathy and exotic plant invasion: from molecules and genes to species interactions. Science, 301, $1377-1380$.

Bennett, J. A., Maherali, H., Reinhart, K. O., Lekberg, Y., Hart, M. M., \& Klironomos, J. (2017). dynamics. Science, 355, 181-184.

Bever, J. D. (1994). Feedback between plants and their soil communities in an old field community. Ecology, 75, 1965-1977.

Heisey, R. M. (1990). Evidence for allelopathy by tree-of-heaven (Ailanthus altissima). Journal of Chemical Ecology, 16, 2039-2055.

160 Klironomos, J. K. (2002). Feedback with soil biota contributes to plant rarity and invasiveness in communities. Nature, 417, 67-70.

162 Link-Pérez, M. A., Dollo, V. H., Weber, K. M., \& Schussler, E. E. (2010). What's in a Name: in a tropical forest. Nature, 466, 752-755. 
169 Packer, A. \& Clay, K. (2000). Soil pathogens and spatial patterns of seedling mortality in a temperate tree. Nature, 404, 278-281.

171 Schussler, E. E., Link-Pérez, M. A., Weber, K. M., \& Dollo, V. H. (2010) Exploring plant and animal content in elementary science textbooks. Journal of Biological Education, 44, 123-128.

174 Uno, G. E. (1994). The state of precollege botanical education. The American Biology Teacher, 56, 263-267.

176 van der Putten, W. H., Bardgett, R. D., Bever, J. D., Bezemer, T. M., Casper, B. B., Fukami, T., et al. (2013). Plant-soil feedbacks: the past, the present and future challenges. Journal of Ecology, 101, 265-276.

179 Wandersee, J. H. \& Schussler, E. E. (2001). Toward a theory of plant blindness. Plant Science Bulletin, 47, 2-9. 
Assign plant \& soil players

No. of plant $\&$ soil players should be equal.

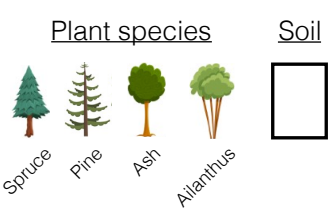

181

\section{Plants ‘condition' soils}

Arrange players in opposing groups \& condition by attaching plants to notecards.

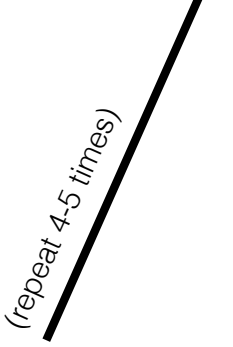

Record diversity

Pause to count species when all plants have survived (TO).
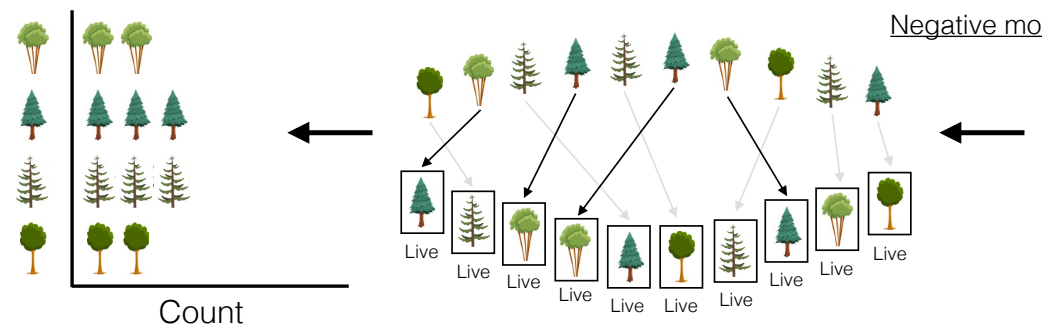

Plant survival depends on species, soil, and mode.

Plants \& soils interact

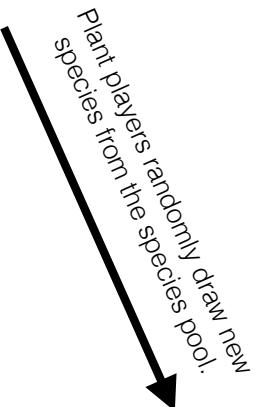

mode
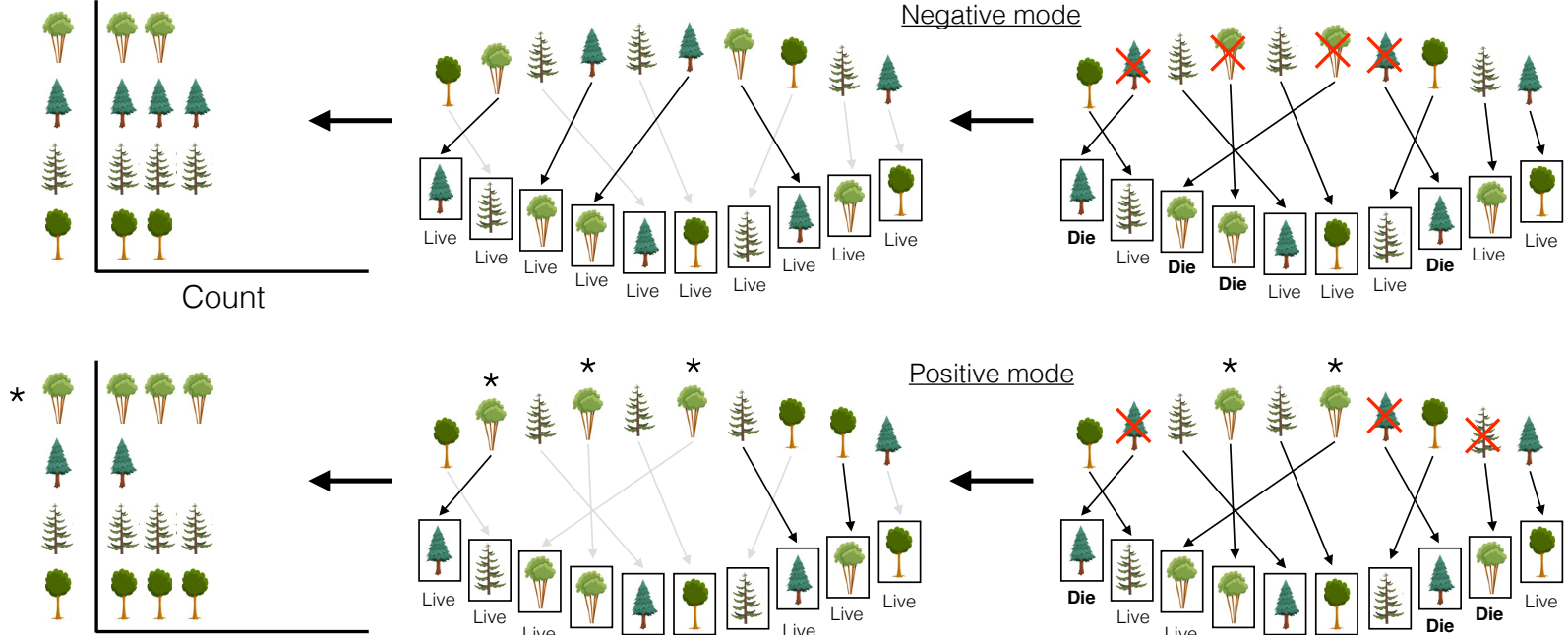

Count
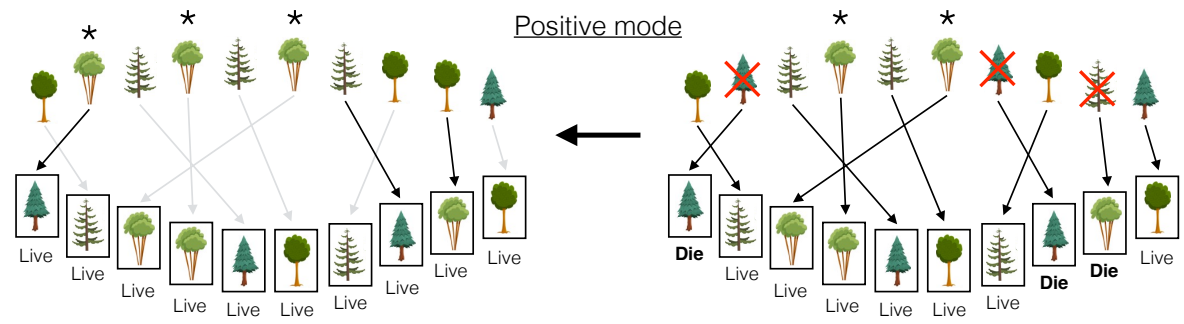

Figure 1. How to perform the activity. (A) The flow diagram outlines the general approach to the activity. The game is based on many random 1-on-1 interactions between plant and soil players. (B) The outcome of plant-soil interactions depend on the mode and identities of both plant and soil players. Plants that live remain matched with their soils (players stand next to one another; grey arrows). Plants that die discard their species, draw a new species from the species pool, and interact with any unmatched soil. In negative mode, plants die when they interact with soils conditioned by the same species (i.e., survival occurs when plants and soils are mismatched). In positive mode, select one plant species that will have positive interactions (e.g., Ailanthus species marked with asteriks). This species survives in all soil types, and no other species can survive in Ailanthus-conditioned soil. Plant species' abundances are recorded once all plants are living to show how the species' abundance and community richness is shaped by plant-soil interactions. 


\section{A}
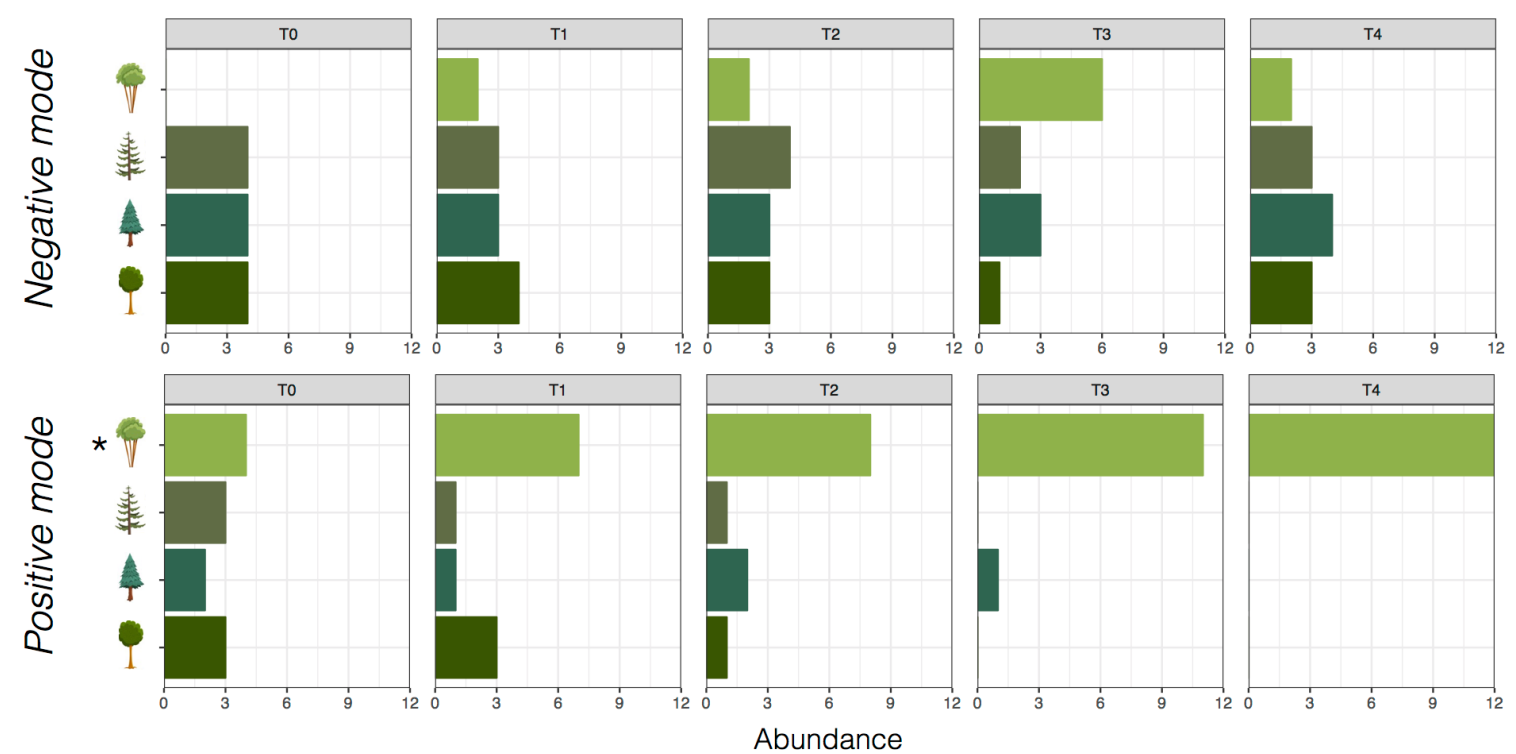

B

194 Pre-activity

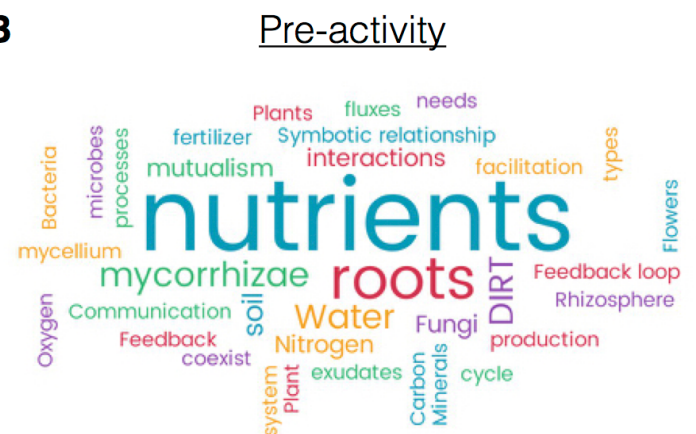

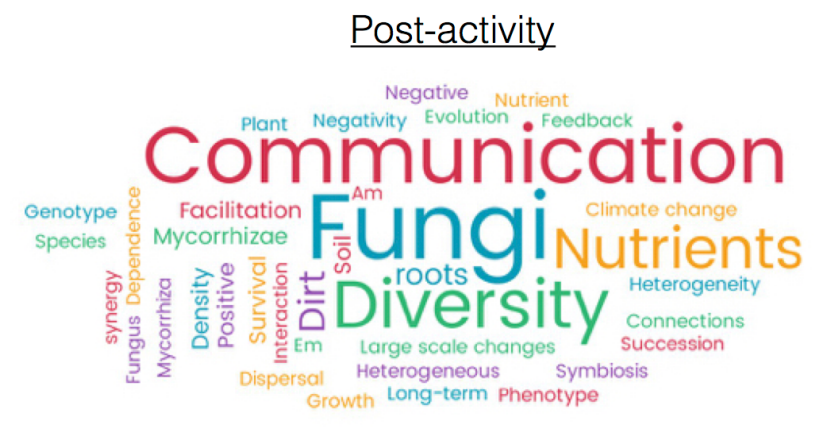

\section{Post-activity}

Figure 2. Evidence that the activity is successful and effective. (A) Modeling plant-soil interactions with the role-playing activity successfully depicted how negative interactions promote community diversity and stability, while positive interactions decrease diversity and disrupt communities. In negative mode, we purposefully set the plant community to have lower diversity ( 3 species) than the total number of species in the species pool (4 species). After one generation $\left(\mathrm{T}_{0}-\mathrm{T}_{1}\right)$, students could see how negative plant-soil interactions increased plant diversity with the addition of Ailanthus into the community. In positive mode, plant community diversity abruptly declined as Ailanthus (an invasive tree species denoted with the asterisk) began conditioning a greater number of soil players that allowed them to persist and inhibited the survival of other species. Results are from a class of 24 undergraduates in an advanced ecology course (diversity was recorded on a whiteboard with the whole class at each time step and broad patterns were discussed when the activity ended). (B) Student responses when asked "What do plant-soil feedbacks make you think of?" pre- and post-activity and discussion. Word sizes reflect their total prevalence in the class responses. Overall, these responses show that the activity improved their understanding of how plant-soil interactions relate to patterns of biodiversity. 